\title{
Gemini: A Non-Invasive, Energy-Harvesting True Power Meter
}

\author{
Bradford Campbell and Prabal Dutta \\ Electrical Engineering and Computer Science Department \\ University of Michigan \\ Ann Arbor, MI 48109 \\ \{bradjc,prabal\}@umich.edu
}

\begin{abstract}
Power meters are critical for submetering loads in residential and commercial settings, but high installation cost and complexity hamper their broader adoption. Recent approaches address installation burdens by proposing non-invasive meters that easily clip onto a wire, or stick onto a circuit breaker, to perform contactless metering. Unfortunately, these designs require regular maintenance (e.g. battery replacement) or reduce measurement accuracy (e.g. work poorly with non-unity power factors). This paper presents Gemini, a new design point in the power metering space. Gemini addresses the drawbacks of prior approaches by decoupling and distributing the $\mathrm{AC}$ voltage and current measurement acquisitions, and recombining them wirelessly using a low-bandwidth approach, to offer non-invasive real, reactive, and apparent power metering. Battery maintenance is eliminated by using an energy-harvesting design that enables the meter to power itself using a current transformer. Accuracy is substantially improved over other non-invasive meters by virtualizing the voltage channel-effectively allowing the meter to calculate power as if it could directly measure voltage (since true power requires sample-by-sample multiplication of current and voltage measurements acquired with tight timing constraints). Collectively, these improvements result in a new design point that meters resistive loads with $0.6 \mathrm{~W}$ average error and a range of reactive and switching loads with $2.2 \mathrm{~W}$ average error-matching commercial, mains-powered solutions.
\end{abstract}

\section{INTRODUCTION}

The need for low-cost, open-source, easily-deployable and installable building-scale energy submetering devices is critically high, as noted by the U.S. National Science and Technology Council [19], the U.S. National Science Board [23], and the U.S. Department of Energy [14]. These reports exist because buildings consume $73 \%$ of the electricity in the U.S. [11] but "no widely-available tools exist to help tenants understand their energy consumption." Submetering devices that meet these requirements and can help address this problem remain elusive as current designs are forced to trade-off between installation ease, accuracy, cost, and maintenance.

Single, self-contained devices [10], [16] require physical contact to the $\mathrm{AC}$ mains in order to measure the $\mathrm{AC}$ voltage signal. This is acceptable for plug-load meters, but ill-suited for circuit level metering inside a panel box. Existing panel metering solutions require a complex installation procedure involving wiring to a spare breaker for capturing voltage and running current transformer leads for each circuit to a central metering device [4]. Panel meters effectively measure at the circuit level but cannot provide insight on a per-load basis. Additionally, these power meters typically use $\mathrm{AC}$ mains and a high voltage AC-DC power supply to power themselves, but requiring a connection to the AC mains makes installation inside of a circuit panel dangerous and expensive. Two recent designs, Monjolo [13] and a piezoelectromagnetic device [25], propose contact-less methods to power the sensors by using energy-harvesting techniques, but both provide limited accuracy because without access to the voltage channel they can only measure apparent power, at best, and certainly not true power. A small, wireless, non-contact, easy-to-install, and accurate power meter would overcome the limitations of current submetering devices.

To address these challenges, we present Gemini, a power metering system that decouples voltage measurement from current measurement to create a non-contact, energyharvesting power meter capable of measuring true power. Two insights enable this design point: 1) a power meter can harvest energy to power itself from the output of a current transformer that it also uses to measure the current waveform and 2) the AC voltage waveform can be virtualized by a single voltage monitoring device [22], and key voltage parameters, instead of actual samples, can be used by the contact-less power meters to compute true power. These insights enable a split metering architecture in which one or more plug-in devices are responsible for measuring and virtualizing the $\mathrm{AC}$ voltage waveform and the majority of the other devices act as power meters that sample the local AC current waveform, locally synthesize the voltage signal, and calculate the power draw of their load (by multiplying voltage and current measurements on a time-synchronized, sample-by-sample basis).

This split architecture permits an energy-harvesting power meter to accurately calculate real, reactive, and apparent power while remaining non-contact, which reduces size, cost, and installation complexity. Gemini can replace a monolithic panel level power meter that requires a control box and wires for every circuit, with small, split-core current transformer-sized devices that can easily be clipped onto circuits of interest. Gemini can also function as a compact plug-load meter to provide plug-load-level submetering. Both versions wirelessly transmit their results to the cloud over a low-power network.

Two central concepts-energy-harvesting and voltage virtualization-enable the Gemini design, but they also add operational constraints not found in other meters. Energyharvesting solves the issue of powering the meter, but it only provides enough energy to intermittently power and activate the device for very short periods of time. To operate under power intermittency, we partition the power measurement task across multiple activations to provide sufficient energy for communications, sensing, storage, and computation. 
Meanwhile, voltage virtualization requires a shared notion of time between the device parametrizing the voltage and the device measuring the current, to properly align the phase of the two signals. Prior work uses clock synchronization protocols, but such approaches are incompatible with the intermittency imposed by energy-harvesting operation. To address this problem, we transfer relative time (or phase offset) using a single packet transmission, allowing the voltage sensor to propagate its local time across domains to the current sensor/power meter's local time.

To evaluate the architecture, we prototype both the voltage monitoring device and the energy-harvesting power meter. We show that virtualizing the voltage channel is a viable approach to designing a power meter. We evaluate the system by benchmarking its performance with resistive, inductive, and switching loads, including ones with dynamic power factors. We find that Gemini can meter resistive loads with an average error of $0.61 \mathrm{~W}$ and inductive and switching loads with an average error of $2.2 \mathrm{~W}$. We also demonstrate that the energyharvesting power meters can harvest and operate with loads as low as $0.5 \mathrm{~W}$. Further, we evaluate the effect of timing in the system and show how timing errors lead to phase offsets and measurement error. Finally, we discuss a technique for matching the correct virtualized voltage channel to the current channel under measurement, describe the trade-offs inherent in the design space, and outline avenues for future work.

\section{RELATED WORK}

A range of commercial and research power metering systems have been designed. Table I compares power meters across three key dimensions: the method used to transmit measurements, the method used to power the meters, and whether the meters can calculate true power (i.e. whether they calculate power by multiplying voltage and current measurements acquired in a synchronized manner on a sample-bysample basis). Only Gemini achieves all three.

\section{A. Plug-Load Meters}

Plug-load meters [1], [3], [7], [10], [16], [17], [21], [24] are capable of providing high-fidelity, accurate power measurements for single loads. However, for loads that are hard to move (e.g. kitchen appliances) or built in (e.g. lighting), attaching a plug load meter is difficult or impossible. Also, plug-load meters are typically active, meaning that they draw power even when the metered load is off. In contrast, Gemini supports plug-load or circuit panel installation, and offers power-proportional power metering (i.e. zero idle power).

\section{B. Whole-House Meters}

An alternative to plug-load metering is whole-house metering in which a single meter monitors the power draw of the entire building. Devices like the The Energy Detective [8] and Blue Line Innovations' PowerCost Monitor [6] provide this data but have high installation overhead and are not capable of submetering. Gemini can also meter whole-house power by attaching to a home's drip-loop or incoming AC line, but it additionally supports load and circuit level submetering.

\begin{tabular}{|l|l|c|c|c|}
\hline & Type & $\begin{array}{c}\text { Is } \\
\text { Wireless }\end{array}$ & $\begin{array}{c}\text { Harvests } \\
\text { Energy }\end{array}$ & $\begin{array}{c}\text { Calculates } \\
\text { True Power }\end{array}$ \\
\hline ACme [16] & Plug-load & Yes & No & Yes \\
\hline Watts Up? net [10] & Plug-load & No & No & Yes \\
\hline WeMo Insight 1] & Plug-load & Yes & No & Yes \\
\hline TED [8] & Split-core & No & No & Yes \\
\hline Magnetometer 20] & Magnetometer & Yes & No & Yes \\
\hline Virtualization & Current transformer & Yes & No & Yes \\
\hline Stick-on [25] & Piezoelectromagnetic & Yes & Yes & No \\
\hline Monjolo [13] & Plug-load, split-core & Yes & Yes & No \\
\hline \hline Gemini & Plug-load, split-core & Yes & Yes & Yes \\
\hline
\end{tabular}

TABLE I: Comparison with prior work. Gemini is unique in that it is wireless, harvests energy, and calculates true power.

\section{Non-Contact Meters}

Realizing that contacting the $\mathrm{AC}$ line for power metering incurs high installation costs and limited deployment scenarios, many recent meters have explored various non-contact options.

1) Non Energy-Harvesting: Patel et al. designed an externally powered house-level stick-on power meter using magnetometers designed to be attached to the circuit breaker in the house [20]. The sensor measures only current, which is later multiplied by RMS voltage by a PC. This split approach is architecturally similar to Gemini but uses RMS values instead of true readings which cause significant error for reactive and switching loads. Also, while unobtrusive for a single sensor, monitoring individual circuits and powering the sensors to do so is challenging and error-prone due to crosstalk from adjacent circuits.

An approach proposed by Schmid et al. is similar to Gemini in that it uses one sensor to parametrize and transmit the voltage channel to another time-synchronized node that is able to synthesize the voltage waveform [22]. The final step of computing power is done offline. Gemini extends this idea by synthesizing voltage, measuring current, and computing power on a single node, by powering that node by energy-harvesting from a current transformer, and by removing the need for a wireless time-synchronization protocol which is challenging to maintain with the limited operating energy budget provided by energy-harvesting.

2) Energy-Harvesting: A piezoelectromagnetic (PEM) device designed by $\mathrm{Xu}$ et al. is both an energy-harvesting device that can power a sensor node and a current sensing device for circuit-level metering [25]. The PEM is a "stickon" device that attaches to each breaker in a circuit panel to meter current. However, the PEM suffers from crosstalk and, without access to the voltage channel, cannot provide true power measurements.

The Monjolo [13] energy meter combines energy-harvesting using a current transformer and energy metering. The device harvests at a rate proportional to the the power of the attached load and converts that rate to a power measurement. This indirect sensing works for fully resistive loads but exhibits inaccuracies for loads with non-unity power factors.

In contrast to these designs, Gemini uses an energyharvesting node to sample the current waveform, wirelessly acquire the voltage parameters, and compute true power. 


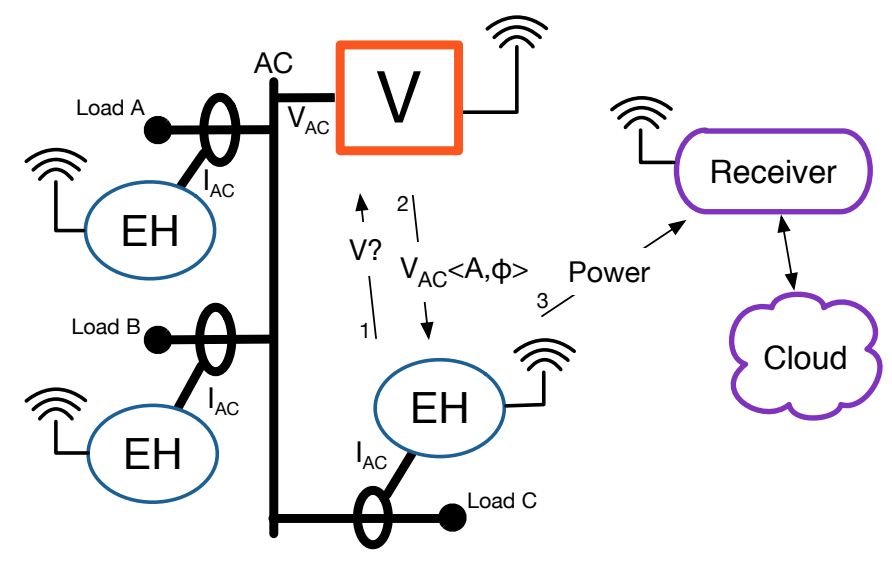

Fig. 1: Gemini overview. A single voltage monitor (V) continuously monitors the voltage channel of the AC circuit. Energy-harvesting power meters $(\mathrm{EH})$ use current transformers to meter each load. To calculate true power, the $\mathrm{EH}$ meter requests the voltage parameters from the voltage monitor, samples the local current waveform, synthesizes the voltage waveform, computes power, and transmits the result to a receiver which forwards the data to the cloud. Maintaining timing across domains is key to the success of this approach.

\section{SySTEM OVERVIEW}

Typical AC power meters simultaneously sample the voltage and current channels and multiply these signals on a sampleby-sample basis to calculate power. Gemini, in contrast, is an $\mathrm{AC}$ power metering system in which the voltage and current waveforms are measured on separate devices and wirelessly recombined to calculate power. This "split" architecture allows for a non-contact, energy-harvesting, true power meter.

Figure 1 shows the two Gemini components, the voltage monitor $(\mathrm{V})$ and the energy-harvesting power meter (EH), which work in tandem to measure power. The voltage monitor continuously monitors the voltage channel and provides a virtualized representation of the voltage waveform to the energy-harvesting nodes on-demand. Each energy-harvesting node measures its local current waveform and multiplies that by the voltage waveform obtained from the voltage monitor to calculate true power. The energy-harvesting node then transmits the power data to the cloud via a receiver.

\section{DESIGN}

Gemini's design employs distributed voltage and energyharvesting current monitors cooperatively calculating power.

\section{A. Virtualizing Voltage}

To realize this split metering architecture, and to successfully calculate power with Gemini, either the current measuring device must learn about the voltage waveform from the voltage monitor or vice-versa. In our design, we transmit information about the voltage waveform to the current sensor to calculate power. The reason for this is that the voltage channel is more easily parametrized than the current channel.

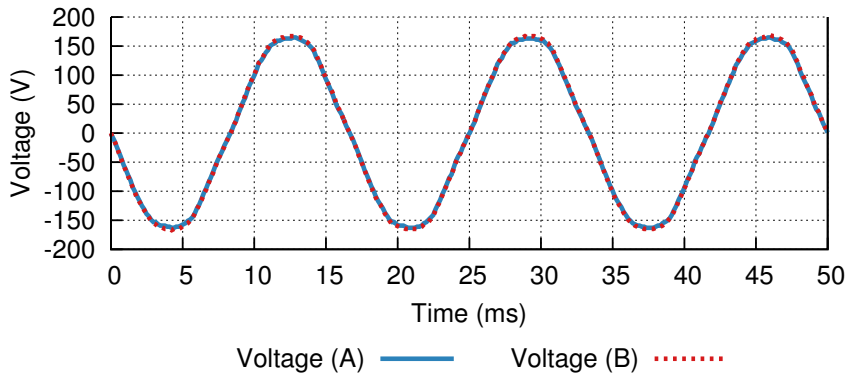

Fig. 3: Comparison of two voltage waveforms at physically disparate points in the same electrical system. Measuring the voltage at two different loads with different power draws on different circuits tapped off of the same leg of a transformer results in voltage waveforms that are nearly identical. This suggests that measuring voltage at a single watchpoint is sufficient for calculating the power draw of multiple loads.

That is, voltage can be modeled with phase information and one or a few Fourier coefficients. Figure 2 provides some intuition for this distinction by showing the voltage and current waveforms for several loads. The voltage waveform is consistently sinusoidal while the current exhibits significant harmonic distortion. Because sending raw waveform samples is infeasible due to limited packet payloads and energy resources, virtualizing the voltage channel and transmitting its smaller parameter set is the preferred approach.

Another tenet of the Gemini design is that only a small number of voltage monitoring sensors are required to support a much larger number of current sensing power meters. This holds if the local voltage measurement can be replaced with a voltage measurement obtained elsewhere in the load tree. To verify this, we sample the voltage waveform at two points on the same leg of a transformer from two different circuits behind two different electrical panels with a $300 \mathrm{~W}$ load on one point and a $1.5 \mathrm{~kW}$ load on the other. The resulting voltage waveforms are shown in Figure 3 The waveforms show no phase distortion and a slight amplitude variation, suggesting that remotely sensing voltage is viable. One other important factor related to voltage measurement is that the phase of the voltage channel local to the power meter (which is not measured) must match the phase of the virtualized voltage channel used for power calculation. A method for matching channels is discussed in Section VII-B.

\section{B. Energy-Harvesting}

To meet the ease-of-installation constraint, the current sensor must be able to sample the current waveform while remaining non-contact. To power the sensor, we are unable to use an AC mains based AC-DC converter because that requires tapping into the circuit, and we do not use batteries because of their size and the maintenance burden of replacing them. Instead, we use an energy-harvesting power supply that uses the output of a current transformer to charge a capacitor. This approach is largely borrowed from the Monjolo meter [13]. 


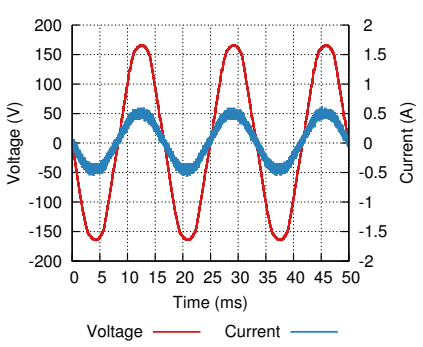

(a) $40 \mathrm{~W}$ Incandescent

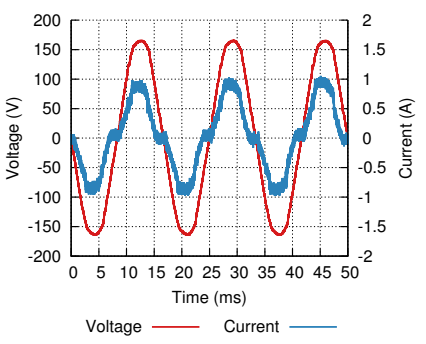

(e) MacBook Pro

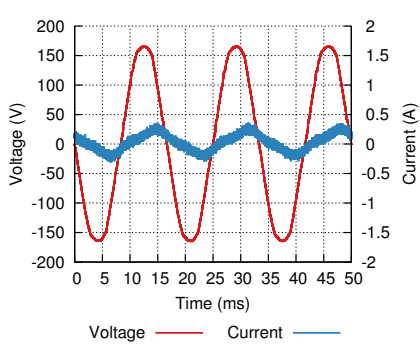

(b) AC Fan

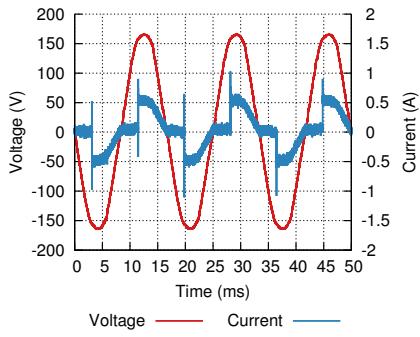

(f) Dimmed Light Bulb

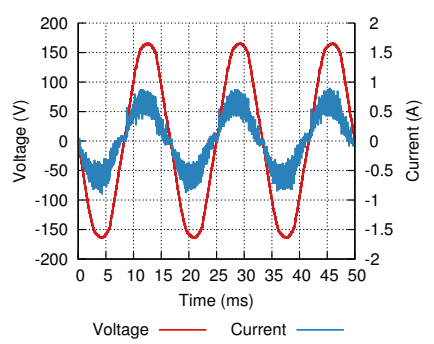

(c) 3D Printer

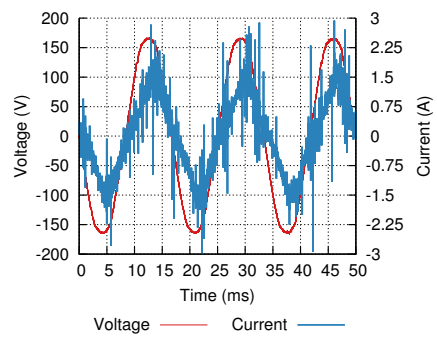

(g) Ice Shaver

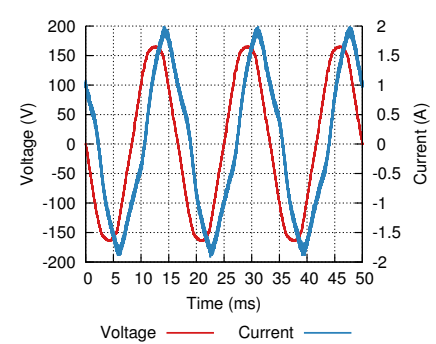

(d) Refrigerator

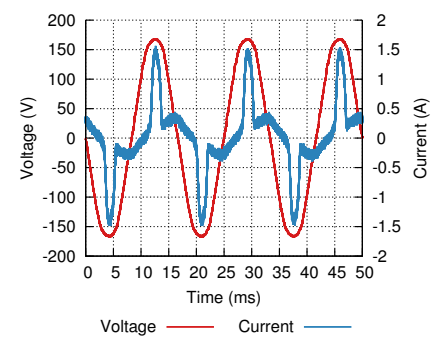

(h) Audio Receiver

Fig. 2: Voltage and current waveforms of eight loads. The current waveforms vary wildly, but the voltage waveforms are consistently sinusoidal and can be represented by one or just a few Fourier coefficients. The sinusoidal nature of the voltage waveform makes it a better candidate to be virtualized, thus supporting our architectural choice to virtualize voltage.

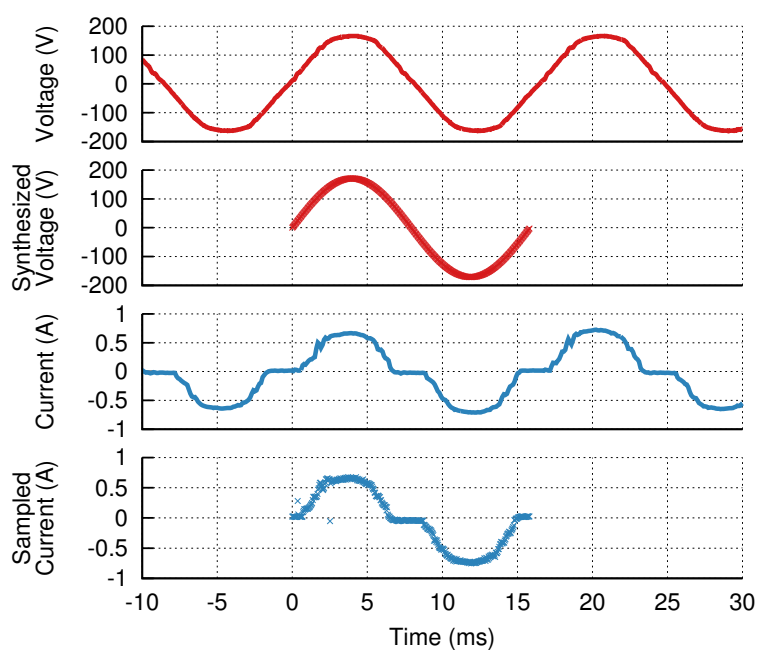

Fig. 4: Voltage and current waveforms. Ground truth current and voltage waveforms are shown above their synthesized/sampled counterparts. The voltage waveform is synthesized from the parameters sent by the voltage monitor and the current waveform is sampled from the current transformer monitoring the load. The discrete waveforms are multiplied together, point-by-point, to compute the load's power draw.

This power supply is necessarily intermittent; harvesting from a current transformer does not supply sufficient power to run a sensor node continuously. It is also unpredictable, as variable-power loads will alter the operation of the harvester. This causes the power supply to limit the sample rate of the meter based on how quickly and often it can recharge.

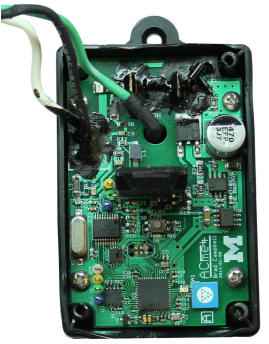

(a) Voltage Monitor

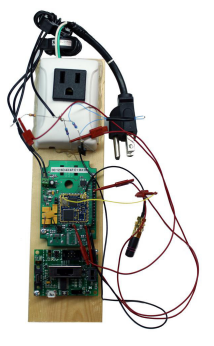

(b) Energy-Harvesting Node
Fig. 5: Sensing hardware.

\section{Power Meter System Operation}

Power is calculated by the energy-harvesting current sensor. The sensor begins by requesting information about the voltage channel from an always-on voltage monitor. After sending the request, it samples the current waveform until the voltage monitor responds. It then synthesizes a discrete representation of the voltage channel properly phase-aligned to the sampled current waveform, as shown in Figure 4. It multiplies the voltage and current samples together and averages them to find the instantaneous power draw of the load. Harvested energy permitting, the current sensor sends the power measurement to a receiver which timestamps and forwards the data to the cloud, otherwise the sample is stored for future transmission.

The receiver is an always-on listener that may be a dedicated gateway for the low-power wireless network or it may be a mains-powered node, such as a voltage monitor, which can forward the packet on behalf of the meter. 


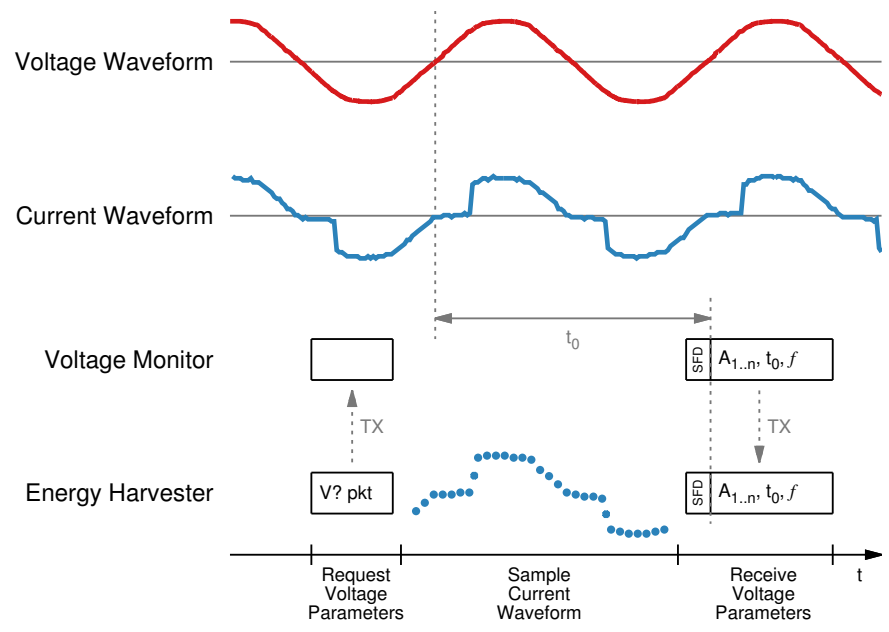

Fig. 6: Transmitting AC voltage phase information. After the energy-harvesting node requests the voltage parameters, the voltage monitor responds with parameters of the voltage waveform such as Fourier coefficients, phase, and frequency. Phase is encoded as the time offset $\left(t_{0}\right)$ between the response packet's SFD signal and the voltage signal's most recently rising zerocrossing. The energy-harvesting node uses its local timestamp of the SFD signal to phase align the current waveform samples to the voltage channel without requiring synchronized clocks.

\section{IMPLEMENTATION}

To evaluate our hypothesis and explore the performance of our design, we implement a prototype version of Gemini.

\section{A. Voltage Monitor}

The voltage monitor continuously records and can ondemand report parameters of its local AC voltage signal. Our implementation uses a TI CC2538 SoC [9] for computation and wireless communication, and an Analog Devices ADE7753 [12] for AC voltage waveform analysis.

The voltage monitor stores the peak voltage of the most recent $\mathrm{AC}$ cycle and locally timestamps the most recent rising zero-crossing of the voltage waveform. This allows the voltage monitor to make a simple parametrization with the phase and magnitude of the voltage waveform for dissemination to interested clients. The ADE7753 also provides access to digital samples of the entire voltage waveform allowing for more sophisticated parametrizing schemes to be used in the future.

The voltage monitor provides the parametrized voltage information as a service. An interested client sends a wireless packet to the voltage monitor requesting the parametrized values. The monitor immediately replies with the magnitude and phase of the voltage signal. Because we do not assume a synchronized timebase between the client and monitor, the phase information is transmitted as a time offset between the start of frame delimiter (SFD) signal of the outgoing reply packet and the most recent rising zero-crossing of the voltage waveform, as shown in Figure 6 The transmitter and receiver establish a common time reference by assuming the SFD signal asserts simultaneously on both devices.

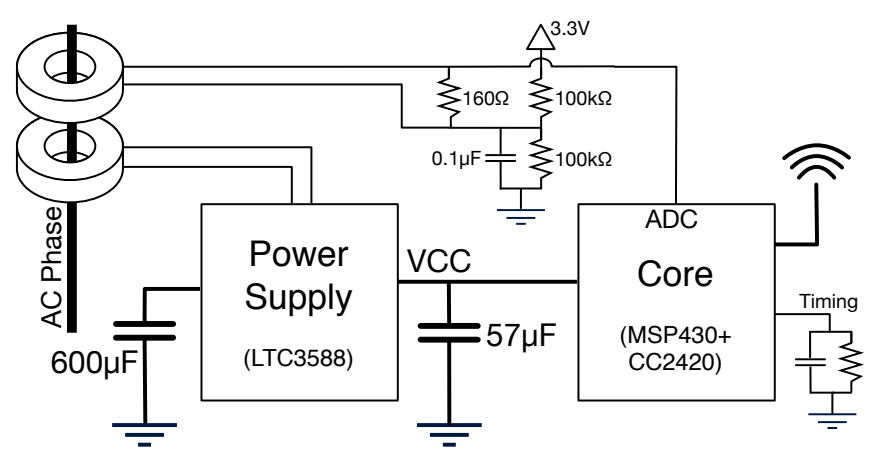

Fig. 7: Energy-harvesting power supply and measurement circuit. One current transformer is used for harvesting and the other for measurement. Future implementations could multiplex one current transformer for both operations.

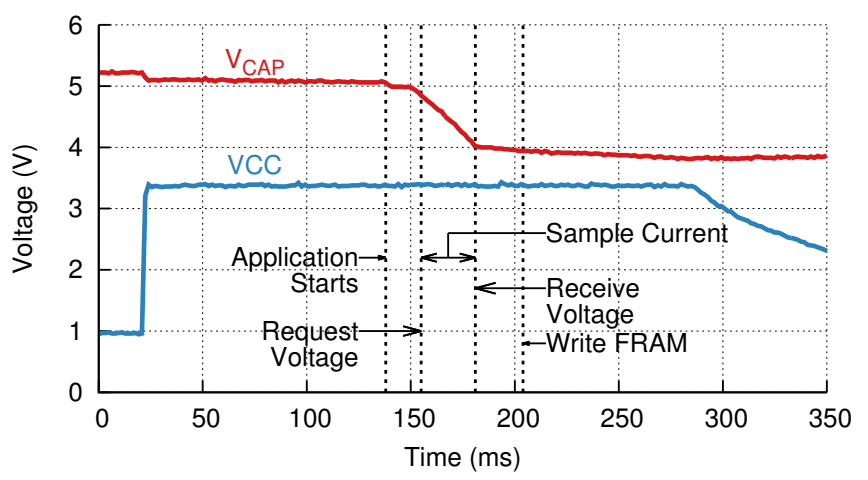

Fig. 8: Energy-harvesting operation. $V_{C A P}$ is the voltage on the $600 \mu \mathrm{F}$ capacitor and $V_{C C}$ is the $3.3 \mathrm{~V}$ supply voltage.

The receiver uses its local SFD timestamp and the rising zero-crossing time offset provided by the voltage monitor to align the voltage and current waveforms. Any delays in packet transmission and processing are ignored because all offsets are calculated from the synchronized SFD signal.

\section{B. Energy-Harvesting Power Meter}

The energy-harvesting device (Figure 7) is responsible for sampling the AC current waveform and calculating the instantaneous power draw of the load or circuit.

1) Power Supply and Coil Circuit: The energy-harvesting power supply is based on the Monjolo power meter's design [13]. The harvesting source is the AC output of a current transformer that is wrapped with 10 windings of the $\mathrm{AC}$ phase line. This AC signal is fed to a Linear Technology LTC3588 [18]. The LTC3588 is configured to charge a $600 \mu \mathrm{F}$ capacitor bank to $5.25 \mathrm{~V}$ and then enable a $3.3 \mathrm{~V}$ output regulator that powers the main system. When the main storage capacitors discharge to below $3.8 \mathrm{~V}$, the regulator is disabled, the storage capacitors begin recharging, and the system runs on the charge stored in the $57 \mu \mathrm{F}$ output capacitor until the voltage drops too low and the system is forced off. One iteration of this cycle is shown in Figure 8 


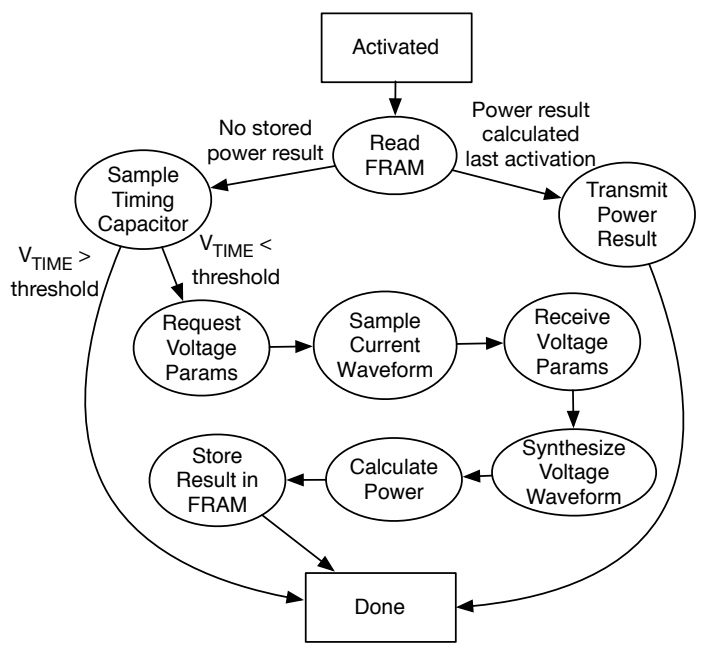

Fig. 9: State machine for the energy-harvesting power meter. Nodes use the timing capacitor to rate-limit sampling and determine when to compute power.

In our implementation, a second current transformer is used for sampling the current waveform. The output of this current transformer is connected across a burden resistor and fed first into a biasing circuit and then into an ADC (shown in the top of Figure 7). The burden resistor affects the scaling factor between the actual current waveform and the current transformer output and increasing the burden resistor increases the peak-to-peak output voltage. To ensure sufficient resolution of the voltage signal from small primary loads we use a $160 \Omega$ resistor. As we will show in Section VI selecting a smaller burden resistor is necessary for larger loads as the ADC saturates. The biasing circuit is required to shift the output of the current transformer from being centered around $0 \mathrm{~V}$ to being centered around $1.65 \mathrm{~V}$. This ensures that all output waveforms up to $3.3 \mathrm{~V}$ peak-to-peak can be successfully sampled by the ADC.

2) Computation Core: The energy-harvesting node is based on the Epic sensor node module [15] which provides a microprocessor and radio. Our design adds a $4 \mathrm{~Kb}$ Cypress FM25L04B FRAM module [2] for persistent storage between activations, and a parallel resistor-capacitor circuit for roughly timing the period of activations to rate-limit activations for high-power loads, as in the Monjolo design [13].

3) Operation: The energy-harvesting node's operation follows the state machine shown in Figure 9 Upon activation, it reads the FRAM to determine if a power measurement was calculated on the previous activation and needs to be transmitted. If so, it transmits the power value to a receiver, transitions to the done state, and waits for the power supply to exhaust. If there is no stored power result, the node samples the voltage of the timing capacitor to determine the approximate elapsed time since the last activation. If the capacitor voltage is above a threshold, the node simply sits idle until the power supply capacitors discharge and the node is forced off again. If the voltage is below the threshold, the node continues operating. This rate-limiting prevents a node from saturating the wireless channel by transmitting packets too frequently. We size the timing resistor and capacitor to limit power calculations to no more than once every five seconds.

When an energy-harvesting node determines that it should calculate power, it requests the voltage parameters from a voltage monitor. After transmitting the request, it begins sampling the current waveform every $40 \mu$ s while awaiting a response from the voltage monitor. The response from the voltage monitor arrives approximately 16 milliseconds after the request, which allows the energy-harvesting node to sample a full period of the current waveform. Upon packet reception, the energy-harvesting node ceases sampling and begins calculating the average power over the prior $\mathrm{AC}$ period.

The response packet from the voltage monitor contains the offset from the SFD signal of the response to the last rising zero-crossing of the voltage waveform in terms of the local clock on the voltage monitor. To convert that offset to the local clock of the energy-harvesting node, the node computes the number of current waveform samples since the voltage zero-crossing. It then uses a precomputed array of sine wave samples to multiply the current waveform samples with the synthetic voltage waveform to obtain the power waveform. The power waveform samples are then averaged to calculate the average power draw. Due to the limited energy budget from the energy-harvesting power supply, the power calculation is stored in FRAM and not transmitted immediately. On the next activation the result is transmitted to the cloud. This imposes a small time delay for the power measurement but allows the system to operate within its energy budget.

\section{EVALUATION}

We now evaluate the viability of Gemini as a power metering system, the effects of using an energy-harvesting power supply on Gemini's operation, and how design decisions and timing errors affect system operation.

\section{A. Power Metering}

To evaluate Gemini's performance as a power meter, we sweep over a range of resistive loads (i.e. loads with unity power factor) and measure how well Gemini tracks ground truth (as measured with a PLM-1LP [5]), as shown in Figure 10. For resistive loads below $5 \mathrm{~W}$ the energy-harvesting meter is unable to accumulate enough energy to activate. Between 5 and $95 \mathrm{~W}$, the estimated power tracks ground truth well with an average absolute error of $0.61 \mathrm{~W}$ and average percent error of $1.2 \%$. Above $95 \mathrm{~W}$ the peak-to-peak output of the current transformer exceeds the $3.3 \mathrm{~V}$ ADC reference and clips, causing greater error. Reducing the value of the burden resistor would allow for these larger loads to be metered.

While Gemini performs well for purely resistive loads (such as incandescent light bulbs), many loads do not have a unity power factor and a purely sinusoidal current waveform. To evaluate Gemini with such loads, we meter four AC loads with both Gemini and a ground truth power meter. The experimental traces are displayed in Figure 11. 


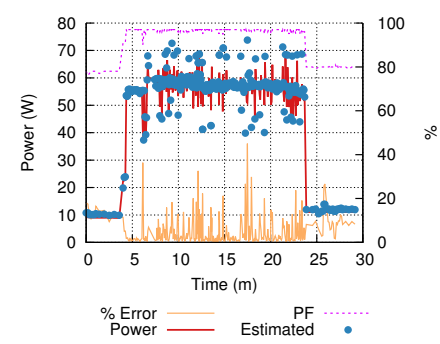

(a) 3D Print Job

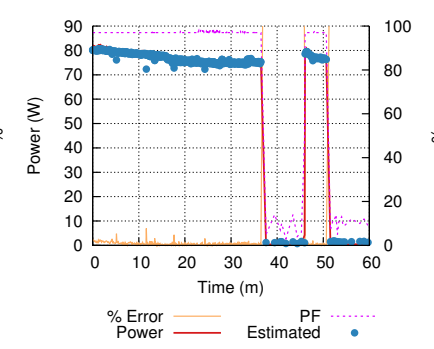

(b) LCD Monitor

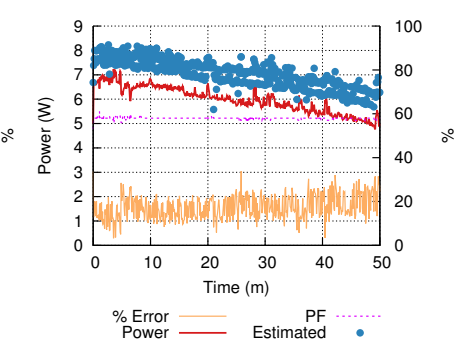

(c) Smartphone Charging

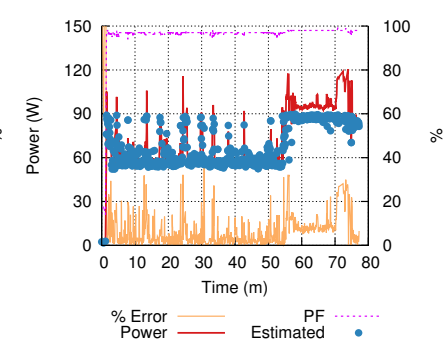

(d) Desktop

Fig. 11: Power metering accuracy for four real-world loads. We meter four loads with a ground truth meter and Gemini. Gemini is able to mirror ground truth well with the highest error appearing when loads either rapidly change power draw or when loads draw very little power (e.g. drawing $\sim 1 \mathrm{~W}$ ). For instance, because (c) has a low power draw, the average percent error is relatively high at $17.2 \%$, but the average absolute error is only $1.0 \mathrm{~W}$. The highest average absolute error is $4.9 \mathrm{~W}$ for (d) mostly due to the current waveform clipping after the 55 minute mark. These test cases demonstrate Gemini's viability as a power meter for real-world loads that operate within Gemini's (configurable) operating range.
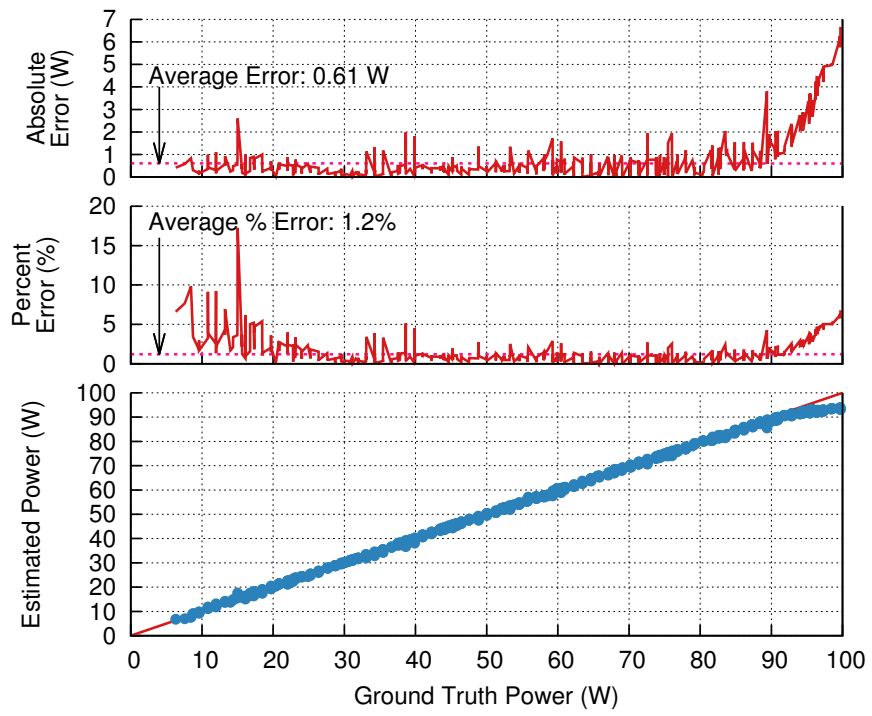

Fig. 10: Accuracy over a range of resistive loads. We meter a range of loads with a unity power factor using Gemini. The $\mathrm{x}$-axis shows the ground truth power draw of the load. In the bottom graph, Gemini's estimate is overlaid on the line representing an ideal device. The middle graph shows the percent error of the estimate, and the top graph shows the absolute error of the estimate. The average percent error is $1.2 \%$ and the average absolute error is $0.61 \mathrm{~W}$. The error grows when the load is greater than $95 \mathrm{~W}$ due to ADC clipping of the current waveform. These results demonstrate that Gemini is a viable power meter for resistive loads.

1) $3 D$ Printer: The printer starts in an idle state before heating the extruder head between minutes 4 and 6 , then actively prints before returning to idle at minute 24 . Gemini tracks these transitions well, even being able to sample the increasing power transition between idle and heating. Rapid swings in the power draw between 50 and $70 \mathrm{~W}$ cause spikes in Gemini's percent error as the energy-harvesting power supply limits the sample rate of Gemini causing spikes to be reported late or missed all together. Overall, however, Gemini reports an average percent error of $4.9 \%$ and an average absolute error of $2.2 \mathrm{~W}$.

2) 24" LCD Monitor: The monitor is cycled between the on, off, on, and standby modes. During the on mode, Gemini is quite accurate with an average error of $0.85 \%$ and average absolute error of $0.67 \mathrm{~W}$. In the standby and off modes Gemini overestimates, but the overall total average error is still only $9.7 \%$ and $0.68 \mathrm{~W}$. A key result of this test is that Gemini continues to operate even when the monitor is drawing only $0.5 \mathrm{~W}$ in the low power states.

3) Charging Smartphone: In this test the power factor is roughly constant at 0.58 . Again Gemini tracks the slowly decreasing power draw well. The average percent error is relatively high at $17.2 \%$, but because phone chargers draw little power, the overall average absolute error is only $1.0 \mathrm{~W}$.

4) Desktop Computer: The final load is a desktop computer that starts in sleep mode, enters normal operation for 55 minutes and then plays video for 20 minutes. At the beginning, the energy-harvesting power meter is able to activate even as the load draws only 1.1 W. During normal operation, Gemini performs well, albeit with the same spikes in error as the load power changes rapidly like in the $3 \mathrm{D}$ printer case. In the final phase of the test, when the desktop plays a video, Gemini underestimates slightly. This is, as in the fully resistive case, due to clipping of the current waveform when sampling it with the ADC. Even with the clipping error, however, the overall average error is $6.3 \%$ and $4.9 \mathrm{~W}$.

For a rough comparison of Gemini's error to commercially available meters, we compare the reported specifications of several of them with the empirical data collected using Gemini. The Watts Up? .Net [10] claims a $1.5 \%$ error, the Kill-AWatt [3] reports a $0.2 \%$ error, and the PLM-1LP [5], our ground-truth meter, cites a $0.5 \%$ error. 


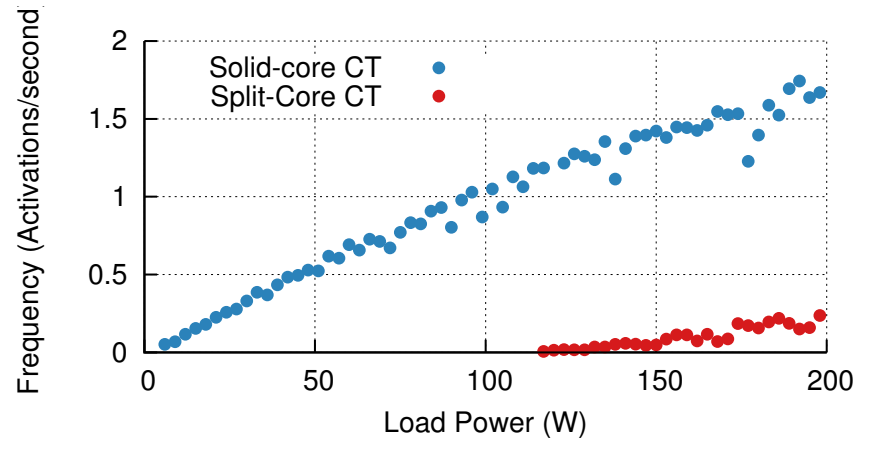

Fig. 12: Activations/second for two coils over a range of resistive loads. The first coil is a solid-core current transformer (CT) with 10 windings of the AC phase line around it. The second is a split-core CT. Below $5 \mathrm{~W}$ for the solid-core and below $117 \mathrm{~W}$ for the split-core the energy-harvesting node is unable to harvest enough energy to activate and therefore is unable to perform any metering. Beyond that, as the load power increases, the ability for the energy-harvesting node to harvest increases. For comparison, a $100 \mathrm{~W}$ load causes the solid-core version to activate at $1 \mathrm{~Hz}$, and because the power measurement is transmitted on the second activation, Gemini offers a $0.5 \mathrm{~Hz}$ sample rate. These sample rates are comparable with other power meters, demonstrating that an energy-harvesting power supply is a viable option.

\section{B. Energy-Harvesting}

A critical element of the Gemini design is the energyharvesting power supply. A consequence of using energyharvesting is that traditional, continuous metering is no longer possible. Instead, power measurements are intermittent with a rate that depends on the power draw of the metered load. To quantify how often an energy-harvesting power meter can perform a measurement, we sweep over a range of power draws using a fully resistive test load and monitor the activation rate of the sensor. We do this both with our main implementation that harvests from a solid-core current transformer wrapped with ten windings of the AC phase line and with a nearly identical implementation that uses a splitcore current transformer around a single wire. The results are shown in Figure 12. For the solid-core version, the energyharvesting node is able to activate once the load reaches $5 \mathrm{~W}$, and it activates more rapidly as the load power increases. At $100 \mathrm{~W}$ the sensor activates roughly once per second, which because the sensor needs two activations to take and transmit a measurement, equates to a maximum sample rate of $0.5 \mathrm{~Hz}$.

For the split-core version, fewer windings mean a higher power load is required for the node to begin activating. At approximately $117 \mathrm{~W}$, the power supply is able to harvest sufficient energy to begin sampling. As in the solid-core design, the sample rate increases with load power. Because the split-core design clips around a wire it is most suitable for metering entire circuits. At the circuit level, the power of multiple loads will sum and the higher minimum measurement power will have less effect than with individual loads.

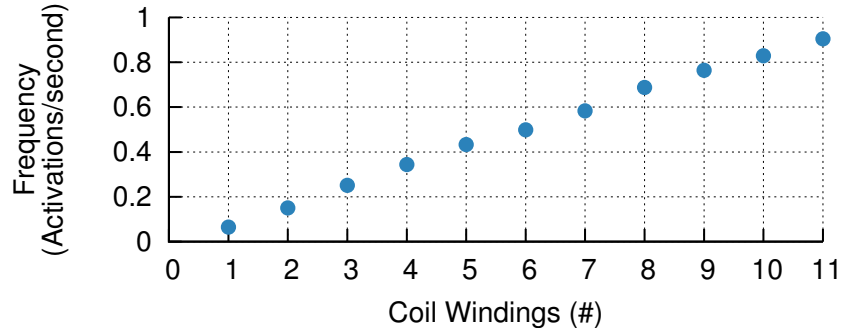

Fig. 13: Effect of the number of coil windings on system performance. The activation rate for the energy-harvesting node with a $75 \mathrm{~W}$ load attached is shown as the winding count changes. As the windings increase, the activation rate, and therefore the sample rate, increases. The increased windings also increase the peak-to-peak voltage output of the current transformer, changing the range of loads that can be successfully measured with a fixed burden resistor.

To further examine the relationship between windings and activation rate we fix the load power at $75 \mathrm{~W}$ and adjust the windings around the coil to observe how the activation rate changes. Figure 13 shows the results. As expected, increasing the windings increases the activation and sample rates. While this aids the temporal fidelity of the meter, it causes the peakto-peak output voltage of the current transformer to increase, reducing the range of loads that the energy-harvesting meter can measure for a given burden resistor. We discuss this, and other trade-offs, further in Section VII-D

One important note to consider is that the data points relating to activation rate with different coils and windings are collected with a purely resistive primary load. In our other experiments from Section VI-A the minimum power required for activation was much lower than $5 \mathrm{~W}$. Based on this, the performance of the energy-harvesting power supply depends not only on the average current the load is drawing, but also on the shape of the current waveform. Therefore, the sample rate will vary based on the power factor of the load being metered.

\section{Impact of Timing Errors on Power Measurement Quality}

The Gemini design hinges on the accurate transfer of timing information about the phase of the voltage waveform from the voltage monitor to the energy-harvesting meter. Timing errors due to clock offsets, clock drift, or queuing delays cause phase errors between the synthesized voltage and sampled current waveforms on the energy-harvesting meter, resulting in power calculation errors. To characterize the effect of such errors, we introduce artificial timing delays by shifting the voltage waveform when calculating the average power of the eight loads in Figure 2. Figure 14 shows power estimation error as a function of the timing error. In the extreme, with $\pm 180^{\circ}$ phase error, or $8.33 \mathrm{~ms}$ timing error, we observe at least $200 \%$ error in the power calculation. This demonstrates that minimizing timing error is critical to Gemini's operation. 

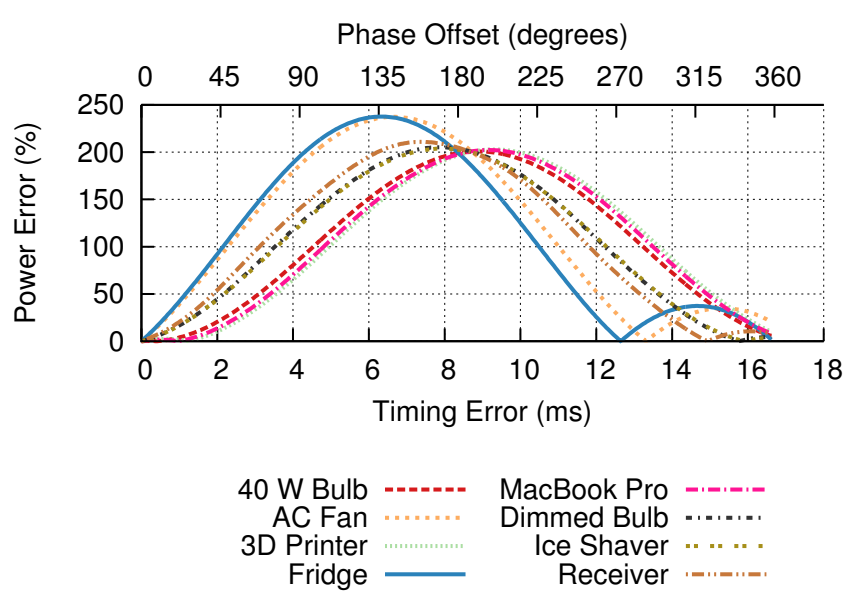

Fig. 14: Effect of timing offsets when calculating power. Even $1 \mathrm{~ms}$ of phase error can result in $50 \%$ measurement error.

\section{DISCUSSION}

In this section, we discuss some limitations of the current design, explore possible workarounds, account for sources of error, and describe inherent design trade-offs.

\section{A. Energy-Harvesting Limitations}

While energy-harvesting addresses issues with how to power the power meter, it has limitations. First, if the metered load is turned off and does not draw any power, the meter does not activate or take measurements. However, a server can interpret the lack of activity as $0 \mathrm{~W}$.

Second, if a load changes power state from active to off (as a refrigerator might do) between Gemini performing a power measurement and it being able to transmit that measurement, the reading could be severely time-shifted from when the load was actually drawing that power. While this could represent a significant error, it is transient and quickly corrected by the next measurement.

\section{B. Matching Current and Voltage}

Buildings typically have two- or three-phase power systems, meaning circuits on different legs of the transformer will have voltage phase offsets between them. In order to correctly calculate power, Gemini must match the correct voltage phase to the load being metered. Loads with a highly displacement based power factor could make selecting the correct voltage channel ambiguous. In practice, however, two factors make this type of error unlikely. First, many loads have switching power supplies which exhibit harmonic distortion to their current waveforms but little displacement. Second, higher power loads are required to include power factor correction which manipulates the current waveform to appear more like a resistive load.

One way we could match the correct voltage channel is to use a simple heuristic: each power meter computes power using all available voltage phases and selects the voltage phase that yields the largest positive power value. Demonstrating this heuristic, we simulate the effect of using incorrect voltage

\begin{tabular}{|l|r|r|r|r|}
\hline \multirow{2}{*}{ Load } & \multicolumn{4}{|c|}{ Calculated Power $(\mathbf{W})$ for V-I Phase Error } \\
\cline { 2 - 5 } & $\mathbf{0}^{\circ}$ & $\pm \mathbf{1 8 0}^{\circ}$ & $\mathbf{+ 1 2 0}^{\circ}$ & $\mathbf{+ 2 4 0}^{\circ}$ \\
\hline \hline AC Fan & 11.777 & -13.286 & -14.932 & 0.588 \\
\hline Dimmed Bulb & 31.991 & -33.184 & -22.175 & -13.406 \\
\hline Light Bulb & 40.564 & -40.087 & -14.745 & -28.346 \\
\hline Audio Receiver & 49.307 & -51.782 & -41.503 & -14.238 \\
\hline 3D Printer & 50.926 & -49.108 & -11.304 & -41.560 \\
\hline MacBook pro & 65.880 & -64.205 & -17.888 & -51.163 \\
\hline Refrigerator & 102.382 & -113.884 & -131.309 & 9.436 \\
\hline Ice Shaver & 104.702 & -107.55 & -71.107 & -44.028 \\
\hline
\end{tabular}

TABLE II: Power calculations with different phase errors. The instantaneous power for the eight loads in Figure 2 is calculated with the current and voltage waveforms correctly aligned and with the current waveform shifted as would happen if the incorrect voltage leg of a transformer was used. In all eight cases the correct result is the greatest positive result, suggesting that using $\max ()$ as a heuristic is often sufficient for correctly selecting the voltage channel.

phases with the loads sampled in Figure 2 The results are in Table II The second column shows the correct result with no phase error. The remaining columns show the calculated power result after subjecting the current waveforms to phase offsets that would occur when using an incorrect voltage channel. For this small sample size, we find the correct phase yields the largest positive value, even for highly inductive loads like the ice shaver and refrigerator.

\section{Sources of Phase Error}

Due to Gemini's distributed nature, several possible sources of error could arise when aligning the phases of the current and voltage waveforms. First, the ADE7753 introduces an average offset of $23.2^{\circ}$ between the rising zero-crossing and when its zero-crossing interrupt line is asserted. We account for this average but additional jitter can cause phase errors. Second, current transformers exhibit phase error offsets and the splitcore version used in Figure 12 adds an average error of $1.5^{\circ}$, for which we must account. Third, quantization errors that manifest from the discrete samples of the current waveform not perfectly aligning with the stored samples of the voltage wave can cause up to $40 \mu$ s or $0.86^{\circ}$ of phase error.

\section{Design Space Trade-offs}

Gemini operates in a rich design space with many axes on which to trade-off different goals. Our implementation chooses one particular design point but helps illustrate how different choices would affect the system. Key properties, like the range of measurable loads, the rate of reporting, and measurement accuracy can all be adjusted. The range of loads that can be measured is a function of the number of windings around the current transformer and the burden resistor. Decreasing the windings or burden resistance increases the maximum load that can be measured. However, decreasing the windings reduces the energy-harvester's ability to harvest, reducing sampling fidelity. 
The sampling rate can be increased by requesting the voltage parameters less often and using the energy spent on the radio to keep a clock running. This would allow a single voltage channel update to calculate several current measurements, at the expense of accuracy due to clock drift. Tuning these properties allows Gemini to be adapted for applications with specific requirements.

\section{E. Future Work}

Several avenues exist to improve Gemini. First, an adjustable burden resistor would allow for a wider range of loads to be metered. Determining the correct value would entail a dynamic search to scale the current transformer output voltage for the ADC reference, or vice versa.

Second, Gemini could improve its voltage waveform synthesis for calculating power. The voltage monitor could compute and transmit additional Fourier coefficients and the power meter could dynamically create the voltage waveform instead of using a precomputed lookup table.

Third, Gemini could be extended to calculate energy as well as power. To calculate energy, a power meter needs to time the interval between power measurements, which it could do by requesting time information from the voltage monitor when it requests the voltage parameters. Then a power meter could multiply the elapsed time by the power measurements to calculate energy. This would be particularly useful for using Gemini as a whole-house meter to corroborate revenue-grade, utility-installed meters.

\section{CONCLUSION}

Existing power meters are limited in their ability to solve the critical problem of electrical submetering in buildings. Installation challenges, maintenance headaches, and metering accuracy hamper the widespread adoption of submetering. To address these challenges, we present Gemini, a new true power meter that offers clip-on installation, energy-harvesting operation, and accurate power measurement. Our work draws on two complementary research themes-virtual sensors and energy-harvesting meters-bringing them together to yield a novel design point in the metering space. Gemini addresses the drawbacks with prior approaches by decoupling and distributing the $\mathrm{AC}$ voltage and current measurement acquisitions, and recombining them over a low-bandwidth, time-synchronized, wireless channel, to offer non-invasive real, reactive, and apparent power metering. This represents a significant advancement from previous designs that are difficult to install, require periodic battery maintenance, or cannot calculate true power. By offering comparable accuracy to commercial meters while vastly improving deployability, Gemini offers a new tool with which to attack the building submetering problem.

\section{ACKNOWLEDGMENTS}

This work was supported in part by the TerraSwarm Research Center, one of six centers supported by the STARnet phase of the Focus Center Research Program (FCRP), a Semiconductor Research Corporation program sponsored by MARCO and DARPA. This material is based upon work partially supported by the National Science Foundation under grants CNS-0964120, CNS-1111541, and CNS-1350967, and generous gifts from Intel and Texas Instruments.

\section{REFERENCES}

[1] Belkin WeMo Insight. http://www.belkin.com/us/p/P-F7C029/

[2] Cypress FM25L04B-DG. http://www.cypress.com/?mpn=FM25L04B-DG

[3] Kill-A-Watt Wireless. http: //www.p3international.com/products/consumer/p4220.html

[4] Obvious Power Panel+. http://www.obvius.com/Products/PPP-O-XX

[5] PLM-1LP. http://www.epd.com/power meters.html\#PLM-1

[6] PowerCost Monitor. http://www.bluelineinnovations.com/powercost-monitor-2

[7] Smarthome iMeter Solo. http://www.smarthome.com/ 2423A1/iMeter-Solo-INSTEON-Power-Meter-Plug-In/p.aspx

[8] TED: The Energy Detective.

http://www.theenergydetective.com/

[9] Texas Instruments CC2538.

http://www.ti.com/product/cc2538

[10] Watts Up? .Net. https://www.wattsupmeters.com/secure/ products.php?pn $=0 \&$ wai $=0 \&$ spec $=1$

[11] Department of Energy (DOE) Buildings Energy Data Book. http://buildingsdatabook.eren.doe.gov/, 2012.

[12] Analog Devices. ADE7753 Datasheet. http://www.analog. com/static/imported-files/data_sheets/ADE7753.pdf

[13] S. DeBruin, B. Campbell, and P. Dutta. Monjolo: An energy-harvesting energy meter architecture. In Proceedings of the 11th ACM Conference on Embedded Networked Sensor Systems, SenSys '13, 2013.

[14] Department of Energy. Building energy efficiency frontiers and incubator technologies (BENEFIT), 2014.

[15] P. Dutta, J. Taneja, J. Jeong, X. Jiang, and D. Culler. A building block approach to sensornet systems. In SenSys'08: In Proceedings of the Sixth ACM Conference on Embedded Networked Sensor Systems, Nov. 2008.

[16] X. Jiang, S. Dawson-Haggerty, P. Dutta, and D. Culler. Design and implementation of a high-fidelity AC metering network. In IPSN '09: Proceedings of the 2009 International Conference on Information Processing in Sensor Networks, pages 253-264, Apr. 2009.

[17] J. Lifton, M. Feldmeier, Y. Ono, C. Lewis, and J. A. Paradiso. A platform for ubiquitous sensor deployment in occupational and domestic environments. In IPSN '07: Proceedings of the 6 th international conference on Information processing in sensor networks, Cambridge, Massachusetts, Apr. 2007.

[18] Linear Technology. LTC3588 datasheet. http://cds.linear.com/docs/en/datasheet/35881fa.pdf

[19] National Science and Technology Council-Committee on Technology. Submetering of building energy and water usage: Analysis and recommendations of the subcommitte on buildings technology research and development, Oct. 2011.

[20] S. N. Patel, S. Gupta, and M. S. Reynolds. The design and evaluation of an end-user-deployable, whole house, contactless power consumption sensor. In Proc. of the SIGCHI Conference on Human Factors in Computing Systems, 2010.

[21] N. B. Priyantha, A. Kansal, M. Goraczko, and F. Zhao. Tiny web services: design and implementation of interoperable and evolvable sensor networks. In Proceedings of the 6th ACM conference on Embedded network sensor systems, 2008.

[22] T. Schmid, D. Culler, and P. Dutta. Meter any wire, anywhere by virtualizing the voltage channel. In Proceedings of the 2 nd ACM Workshop on Embedded Sensing Systems for Energy-Efficiency in Building, pages 25-30. ACM, 2010.

[23] U.S. NSB. Building a sustainable energy future: U.S. actions for an effective energy economy transformation, Aug. 2009.

[24] T. Weng, B. Balaji, S. Dutta, R. Gupta, and Y. Agarwal. Managing plug-loads for demand response within buildings. In Proceedings of the Third ACM Workshop on Embedded Sensing Systems for Energy-Efficiency in Buildings, pages 13-18. ACM, 2011.

[25] Q. Xu, I. Paprotny, M. Seidel, R. White, and P. Wright. Stick-on piezoelectromagnetic AC current monitoring of circuit breaker panels. Sensors, 13(3), 2013. 